\title{
GASTROMANIAC. HOW (FOOD) INFLUENCERS CREATE TRUST
}

\author{
Kristian Bankov \\ New Bulgarian University \\ kbankov@nbu.bg
}

\begin{abstract}
In the first part of the paper, I shall offer a brief overview of a hypothesis developed in another publication, which explores the relationship between the primordial feeling of trust that each person's face elicits to varying degrees and how this represents a type of capital for influencers. In the second part of the paper, I shall develop this model using theoretical know-how from the field of brand management, where a beneficial link between the influencer communication model and that of legendary brands emerges. Thus, for an influencer to build invaluable trust capital with his/her followers in the first place, he/she must start from the position of some passion or sacred beliefs which give authenticity to the core expertise underlying the influence being exerted. In this model, the communicative performance of the influencer and the quality of his/her narrative take centre stage. Credibility depends on the synchrony between these elements and the extent to which the constructed public influencer's character is true to itself in its various manifestations. An explicitly or implicitly defined lifestyle is always
\end{abstract}

Digital Age in Semiotics \& Communication, Vol. III-IV, 2020/21, 42-56 
present in the system. It provides concreteness and makes it easier for followers to compare and imitate.

Keywords: influencers, trustworthy face, suspension of disbelief, narrative, mythology

\section{Introduction}

In this study, I aim to apply a semiotic approach as a key to understanding the extraordinary success of food influencers in the context of the overall epic of gastromania during recent decades. As a starting point, I shall take the research I have already conducted on the complex relationship between the face and trust-building process of influencers - the new universal meta-profession - and their followers (Bankov 2021). As we shall see, face is a very important prerequisite for the success of any influencer. However, the overall mechanism for making an impact is much more complex and involves a permanent performance. The ultimate goal and at the same time the driving force is trust. The main inspiration for articulating the components of this 'influencer system' is the system of brand mythology as articulated by Vincent (2000: ch. 1). Only after defining this system can we delve deeper into the specifics of food influencers to see exactly how the trust - suspension of disbelief relationship fundamental to their success works for them.

\section{Energy and the Trustworthy Face}

Despite the large amount of semiotic research carried out, especially in recent years, the face remains an unresolved semiotic enigma. It can be compared to a volcano in which the daily eruption of meaning is easily observable, even though the energy causing it in the bowels of the earth may not be visible. One very important aspect of this exploratory universe is the issue of the trust that people build up in the course of social interaction, based on the face. A number of authors believe that the instantaneous judgment of whether or not to trust a person is primordial and pre-rational. There are even scientifically identified centres in the brain (amygdala) where this judgment arises spontaneously (Colins 1998). This would imply that we construct our entire communicative strategy with respect to a new interlocutor on the basis of this primordial judgment. However, as we will see in the second part of the paper, this is subject to validation or rejection in the course of communication. 
In the cited article, I define the semiotic function of this primordial trust in terms of communicative effectiveness by analogy with the principles of theatrical anthropology for persuasive stage presence. According to Eugenio Barba (1993), the founder of this discipline, there are universal principles of an actor's stage technique which absolutely regardless of the tradition he/she belongs to and regardless of the role he/she is playing, make it possible to capture the spectator's attention. On the contrary, if he/she does not respect these principles, the work may be unspectacular and boring, no matter how great. These principles are derived from the contrast between the daily and extra-daily mode of behavior of the actor's body and psyche. If the principle of our everyday pragmatic motor behaviour is to achieve the maximum result with the minimum motor effort and to routinize every repetitive operation, on stage it is the opposite. Whether it is a dramatic, dance or ethnic performance, every action on stage is characterized by an excess of energy - maximum effort for minimum result. This over-expenditure of energy, when harnessed through a particular (and whatever) theatrical technique, becomes an expressive force, a stage impact. The welltrained and talented actor possesses this. He/she is always convincing, no matter what the role played on stage, and the spectator always believes him.

Similarly, some influencers' faces are persuasive and activate instant trust. Others do not have this impact, completely regardless of the subject of the videos. Both become influencers, but, as Plato would say, fate has stamped the faces of one on gold coins, and of the other on bronze. To illustrate observations guided by the same idea, albeit in a different theoretical framework, a team from PAPER magazine synthesized the faces of the hundred most significant influencers to show the ideal one, i.e. the purest gold coin (Fig. 1).

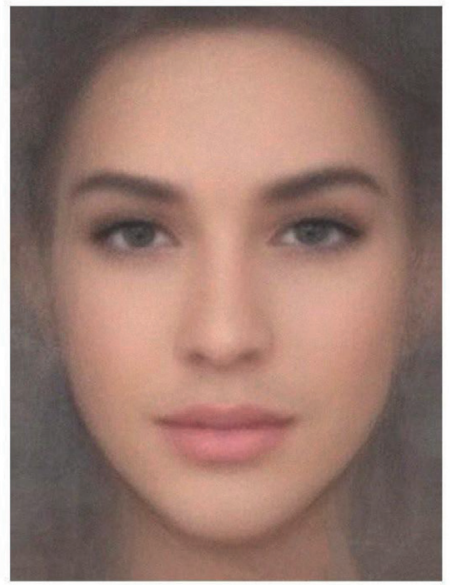

Fig. 1: The Ideal Influencer (Synthetic face of the 100 top Instagram influencers credits to PAPER Magazine/ PAPERMAG.com) 


\section{The Influencer (Mythology) System}

In theatre anthropology, Barba develops the concept of the South Pole actor and the North Pole actor. The former is a native talent who invents the rules of his own technique (because there is no impact without an established technique that makes the play consistent), while the latter is a well-trained actor in some strictly codified technique such as classical ballet and No theatre. One could certainly develop further analogies to the techniques of influencers here, but I will refrain from doing so here, since I think analogies in the other direction are more productive.

We are still talking about influencers in general, not those devoted to gastro themes. At first glance, it would seem a stretch to say that every influencer is a micro brand, but that is the direction I find most forward-thinking. You may react immediately by saying that influencers like Huda Kattan and Elle Darby have created macro rather than micro brands, and I would not say anything against such a statement. However, that is not the idea. These influencers have broken into the public domain as such and have subsequently chosen to capitalise on their fame and expertise in the brand form.

This is also the case with food influencer Sophie Yotova, as we will see in part three. Many other lesser-known influencers, on the other hand, create their own brand elements such as name, logo and video splash before they commercializing their influence capital. In many cases they may not ever have such an intention.

I am talking about something else. In his extraordinary book Legendary Brands (2002), Lawrence Vincent develops a dynamic theoretical framework to distinguish the omonimous types of brands from ordinary product brands. My thesis in this part of the study is that the mechanism by which influencers create their influence is something like a simplified but very essential replica of the mechanism of legendary brands, something akin to its shortcut. So let us see exactly what this mechanism looks like.

According to Vincent, legendary brands engage their followers much more with their storytelling and symbolic equity than with the functional benefits of the products and services they sell. This happens when these companies put into circulation a brand mythology system, instead of throwing all their efforts into the classic marketing mix. This system is precisely a kind of symbolic performance in need of constant renewal (Fig. 2). 


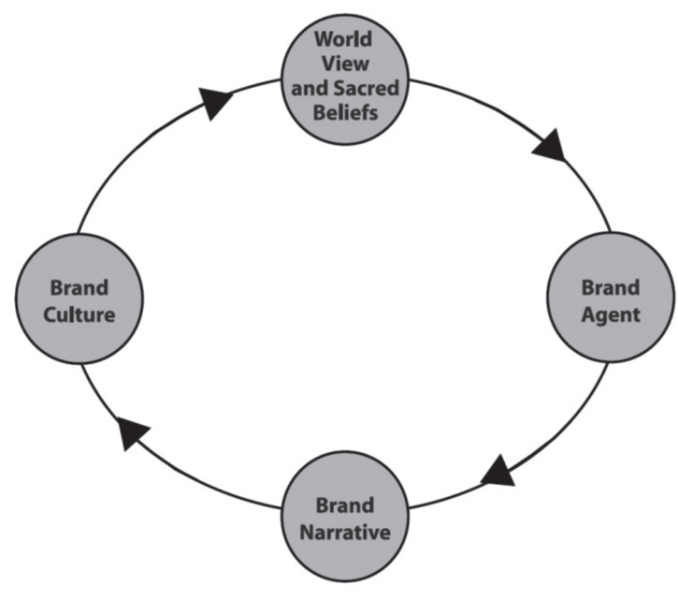

Fig. 2: Brand Mythology System

The genesis of this performance is rooted in the worldview and sacred beliefs of the creators of the brand. It is most often a visionary like Steve Jobs, Richard Branson or Elon Musk. However, it can also be a manifesto with no prominent personality behind it, or a cult status that emerged from consumers, which the company then turned into its own mission and sacred beliefs, as happened with Harley Davidson and Coca Cola. The analogies with religious faith are many and well developed by the author. It is important thing to stress that these are two parallel worlds, and the purpose of the analogy is primarily to highlight the power of the impact of legendary brands.

Worldview and sacred beliefs are not in themselves impactful. They must be put into circulation by a Brand agent. This is done most effectively by the visionary founder of the company himself, but practice knows many other cases. A Brand agent can become some extremely popular endorser celebrity, such as Michael Jordan for Nike or Luciano Pavarotti for Lavazza. The product itself can acquire cult status and thus become a brand agent - an emanation of the brand's values, like Harley Davidson's muscular motorcycle or Coca Cola's bottle. Brand agents are the material proof that the brand's worldview and sacred beliefs are real.

Brand agent, however, is not a silent statue. It speaks and broadcasts messages through many different channels. This is not indiscriminate content, but a highly impactful and coherent narrative which from many perspectives is the most important element of the entire brand mythology system. It is in the context of the brand mythology system that Vincent emphasises that a strong story can evoke a willing suspension of disbelief in its addressee. Thus the addressee leaves the critical and practical everyday 
Self and becomes transported into the fairy-tale world of the brand, where its worldview and sacred beliefs are best embodied (Vincent 2002: 32). This is exactly what does not happen with ordinary product brands, which on the contrary play it safe and direct all their efforts into winning over the pragmatic everyday Self with more functional benefits, price, delivery, etc.

This confrontation is also relevant to our overarching theme, namely building trust. In both types of brands, business success is built on trust. In the former case it is built with the narrative, while in the latter with the qualities of the product or service. Here we must not fall into the naive fallacy that good narrative can sell all sorts of poor products, and that it is possible to do business without any aestheticized communication. While in the latter case the process of trust-building is quite obvious and well described by marketers, it is from a deeper analysis of the former mechanism that the most interesting findings for this research emerge. The relationship between the willing suspension of disbelief and trust is perhaps the only important point left undeveloped by Vincent in his otherwise brilliant book.

Norman Holland, one of the most thorough researchers of the concept of the willing suspension of disbelief in the context of art reception, postulates and proves a direct link between this process and trust. According to him, our ability to switch our everyday identity into another fictional one is a regressive process rooted in the infant's primordial moments of development when completely dependent on the mother. He refers to Erik Erikson's notion of basic trust: "Basic trust in mutuality is that original "optimism," that assumption "somebody is there," without which we cannot live" (Erikson in Holland 1967: 3). Further "We can "lose our selves" in a story, for art takes us back to that point in our development at which we first became aware of our own identity through a trust in nurture and feeding." (5) Human insatiability for experiencing fictional stories is an extension of the childhood insatiability for nurturing and attention, especially when these are associated with the pleasure of feeding. "It is precisely our expectation of pleasure that makes us treat what we know to be fiction as a real experience. It is precisely our ability to treat the fiction as a real experience that enables us to take pleasure from it." (7).

Although brands may not offer as pure a form of transference from the real to the fictional world as literature does, there is no doubt that many of them engage numerous consumers with their inspiring narratives. Some even ostentatiously identify with the brand, especially in social media terms (Bankov 2018). Such practices bring us to the final element of the brand mythology system - brand culture. It can be both real and wishful 
thinking. If a brand is compelling enough to succeed in imposing its products or services into the consumer rituals of individuals, as well as their families and communities, it forms a culture. If it is all well-told and put into circulation in the public domain, but has not yet actually made an impact, it is a wishful brand culture.

In comparison, we can conditionally say that ordinary product brands aim to enter the routine of their consumers, into their practical everyday life where they are looking for a way to make their lives easier and more economical. This routine/ritual opposition can also be seen as a division into two macro axes of consumption. The first of these is oriented towards saving time, while the second is oriented towards filling saved time with meaning/value/culture. Washing machines, vacuum cleaners, household chemicals, drills, frankfurters, mattresses, wardrobes, microwaves, organisers, home robots, inter alia, are the usual industries which satisfy the first type of consumption needs. On the other hand the industries of entertainment, hobbies, sports, alcohol, fashion, gourmet, tourism, design, jewellery, perfume, luxury, experiences, social media, etc. are of the second type.

It is impossible to draw a clear dividing line between these industries insofar as almost every product category has brands priced as practical, and others which claim to be luxury or utopian (Floch 2001: Ch. 5). However, we can say with certainty that the brand mythology system works better in the second type of industries, those oriented towards filling time with meaning and culture rather than the utilitarian and time-saving.

If we now move into the realm of influencers (but not yet into the gastro sector), we find that, similar to brands, there is also a tremendous thematic diversity that can be most conventionally grouped in a similar way of time-saving and time-filling practices. Time-savers, for example, are the so-called "How to do" influencers who offer easy solutions to almost any everyday problem, while time-fillers are all influencers from the world of cinema, sports, fashion, etc. The question here is whether there is a difference in the mechanism of trust creation between influencer and follower in one case and the other, as there is with both types of brands and their consumers.

According to the perspective of this study, the influencer system, regardless of the category of practice, is almost entirely analogous to the brand mythology system. We shall not count as influencers channels presented by content creators whose face does not figure in photos, nor a first-person voice in the videos. There is such a broad category of anonymous practical problem-solving channels which, albeit widely followed, cannot be counted in the category we are interested in. 
As already mentioned, the trust-building system of influencers is structured in an identical way to that of the brand mythology system, although more immediate, more effective and on a smaller scale. In Fig. 3 I have tried to articulate its analogous elements:

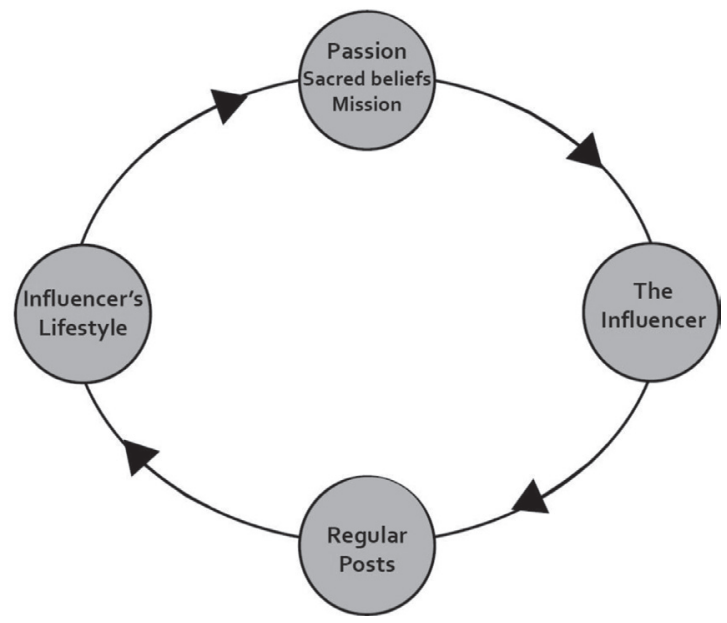

Fig. 3: The Influencer's System

Let us start with the fact that the basis of both systems is trust and that they are most often linked. Without committing ourselves to exact statistics, I can confidently say that most influencers create content on the back of brands, as satellites to already established brand mythology systems. In turn the companies behind the brands use influencers to reinforce that same system. This is exactly the idea of influencer in the eyes of marketing people: "It is important to note that these individuals are not merely marketing tools, but rather social relationship assets with which brands can collaborate to achieve their marketing objectives." One of the best pieces on the subject develops the possible meaning of these social relationship assets in the context of contemporary culture:

Influencer culture, as we know it today, is inextricably tied to consumerism and the rise of technology. The term is shorthand for someone (or something) with the power to affect the buying habits or quantifiable actions of others by uploading some form of original-often sponsored-content to social media platforms like Instagram, YouTube, Snapchat, or, god forbid, LinkedIn. [...] True influencerdom presupposes a particular type of relationship between content creator and viewer, at scale, one that hinges

\footnotetext{
${ }^{1}$ https://influencermarketinghub.com/what-is-an-influencer/ (accessed 3 August 2021).
} 
on the willingness of the viewer to be influenced. (my italics, Martineau 2019)

If the driving force of the brand mythology system is often global ideas, beliefs and worldviews related to the future and well-being of humanity, freedom, love and creativity, then the driving force of the influencer system is most often a passion for doing something (and never a necessity or obligation) inspired by these ideas put into circulation by the brand mythology system.

Influencers most often start out as passionate and expertly enlightened fans who volunteer to take on the mission of explaining and convincing other fans and potentially interested audiences about how great their favourite brand, sports club, filmmaker, travel destination, or culinary experience is.

Over time, this mission develops an autonomous reputation for expertise across the entire product category, and so the influencer begins to gain a growing following.

What replaces the brand agent and makes the influencer system most effective is his/her constant living presence in the process of influencing followers. With brands, this is more cumbersome, although more and more companies are adopting influencers' best practices. The living and immediate presence of the influencers' personality enables the identification of his/her constructed character with the effectiveness of cinema and literature. Many influencers construct their character by talking openly about traumas and complexes from their past and childhood that are not usually talked about, even though the majority of people are affected by them. Thus, Huda Kattan cleverly weaves in self-ironic jabs about her pubescent facial issues when launching her beauty product. Sophie Yotova, as we will see in the next section, introduces her unique technique of healthy cooking as a way of dealing with the complete loss of her sense of smell in childhood. These are indispensable factors in building the confidence that underpins the whole system.

The influencer achieves all this with narrative. A good influencer is a good storyteller. According to the model proposed here, narrative skill can compensate even for a deficit in the primordial confidence emanating pre-expressively from the influencer's face. As we saw in the previous chapter, the primordial instance of "basic trust" establishes the relationship between fiction and the pleasure principle, rendering the willing suspension of disbelief very easy and desirable.

Although from a position of quite different premises, Martineau refers to an analogous instance of the influencer system, namely "the willingness 
of the viewer to be influenced" (my italics, 2019). While at first glance it may seem far-fetched to claim that the influencer narrative is a form of fiction that requires a corresponding disposition on the part of his/her followers, on reflection this is precisely where the difference lies. It is between one who purposefully influences consumer choice and those who, anonymously hidden behind data, provide objective information, leaving the responsibility for consumer choice to the reader/viewer. The figure of the influencer is a product of contemporary digital consumer culture, where big brands fuel the collective imagination with vast amounts of creative content. The influencer is an expert seller of experiences in this universe of meaning. To successfully sell these expert experiences he/she builds $a$ character. The more authentic, immediate, and true that character is, the greater the prerequisites for invaluable credibility. This character, however, has a very labile relationship with the real personality of the influencer. It costs effort in the production of content, just as effort requires first the work of the playwright and then the work of the actor upon the role. This does not prevent us from seeing some influencers so absorbed in their public role that their real personality (and sometimes life) remains almost in the shadow of it, just as some great actors have been said to be much more real on stage than off.

The influencer is not a great artist, but an authentic form of symbolic life that has emerged spontaneously in the conditions of the free market, digital capitalism and consumerism.

The influencer is a fictional character, which is what more and more "ordinary" people are becoming, insofar as maintaining our online identities makes it impossible to resist the temptation to pretend to be better, prettier, healthier, happier than we actually are. The viral nature of the classic trickle-down effect, whereby members of digital society imitate, compete with and therefore influence each other (Volli 2016: 59) affects the majority of modern people. What distinguishes the influencer from the rest is the deliberate effort to bring about some result, be it monetary or symbolic. In this situation, also characterized by fake news and hyper-transient values, a large deficit of reference points for a meaningful life is formed. Therefore, the element that closes, so to speak, the influencer system is lifestyle. It is always present, explicitly or implicitly. Using the theatrical analogy, it is the staging (set design and direction). Here we define lifestyle as a sum concept related to the illustration of the expert or euphoric experience with which the influencer creates value for his/her followers. This exemplification is never out of context. It occurs most often in the influencer's home, technology or art studio, and very often involves active interaction with the 
environment. The lifestyle makes the influencer's entire system more easily accessible for influence and imitation.

\section{The Particular Case of Food Influencers}

I believe that the model described so far fully reflects the mechanism of influence used by food influencers. At the same time, I cannot help but make an observation that fits into the general context of the gastronomic mania which has unfolded with particular magnitude. Does the fact that basic trust is built on nurturing and feeding give this topic a privileged position in building influencer-follower trust? Can we say that in the particular case of food influencers, the suspension of disbelief returns home? Our lives depend much more on what we ingest as food than on the clothes we wear, the make-up we apply or the sports we play. Could it be that the dietary practices passed on in this influencer sphere require a qualitatively different level of trust to those in other areas? At this stage, I would be unable to provide answers to these questions with any academic confidence, but I am convinced that interesting future research can be developed in this direction.

I referred to two case studies of influencers, on the one hand radically opposite, on the other structurally quite similar. The first one is one of the world's most famous food influencers, Harley Morenstein, and the second one is the successful Bulgarian health cooking specialist Sophie Yotova.

Harley Morenstein is a Canadian actor and thrash artist, creator of the culinary YouTube channel Epic Meal Time ${ }^{2}$ with seven million followers, where he built the character Sauce Boss. Since 2008 he has been uploading videos of food excesses that are unacceptable to good taste and common sense. On the videos he is always with friends, consuming alcohol and necessarily eating the unhealthy and sometimes dangerous food that he previously spectacularly prepared. Morenstein's style of character performance is very similar to that of wrestlers.

Morenstein is a man of show, action and vision. He does not use many written words to pick apart a ready description of his influencer passion and sacred beliefs. Still, the YouTube channel's short description is telling: "We make your dreams come true... and then we eat them.." The Facebook slogan is similar: "Where epic meals come to die... ". A much more specific wording is found in the description of the video game EpicMealTime created after the huge success of the show. Its goal is to feed Morenstein's av-

\footnotetext{
${ }^{2}$ https://www.youtube.com/channel/UCYjk_zY-iYR8YNfJmuzd70A (accessed 18 August 2021).
} 
atar with as much fatty, calorie-dense and unhealthy food as possible, and allowing the character to eat a vegetable or other healthy product results in a loss of life.

What has given rise to this passion for unhealthy eating and excess is the general context of the healthy eating and veganism boom of the last 1015 years, which have imposed a sort of nutritional "political" correctness worldwide. EpicMealTime is a space for food and eco incorrectness.

Sauce Boss is a vivid character that is instantly memorable and leaves no one indifferent, a true brand agent of EpicMealTime. Quite a few of the comments below the video are of disgust, but of course fans prevail. The character is convincing and authentic. This is backed up by a very realistic display of the physiological reaction to Morenstein/Sauce Boss that follows trying the "World's spiciest pizza" or a Snickers wrapped in bacon and fried for several hours in a skillet.

Morenstein's narrative is produced once a week. It employs intense editing, rudimentary animation and sound effects to enhance the "epic" moments of the culinary performance. Perhaps the key to the impact is found in the fact that any viewer with the appropriate mindset would be curious to see how the world's spiciest pizza is made and especially bitten off, or how a roast pig is stuffed with sausages that have previously been wrapped in bacon and thoroughly fried, or how a UFO burger and cheeseburger baklava are made. Morenstein is extremely creative. From any food topic he can produce culinary absurdity. McDonald's is often the subject of similar absurdity, when an entire menu, for example, is stuffed and cooked like a burrito, or when a Big Mac is wrapped in a ridiculous amount of bacon and fried for hours. The archives show burger towers with 50 meatballs or pizza topped with burgers, covered in mozzarella and baked in the oven.

His manner of storytelling is immediate and spectacular at the same time. In many cases he takes the scene to supermarkets where the whole group is happily shopping. At other times they visit interesting restaurants and eat and comment on dishes unique to them. Morenstein is identical when it comes to interacting with both casuals and fellow diners.

All of these narrative moments embody the passion and beliefs of the influencer in a distinctive lifestyle that is easy to imitate and influence. Eating is the most effective element of our daily life to ritualize, thus energizing it with meaning in addition to the nutrition. Morenstein is extremely consistent and always true to himself in the "nutritionally" incorrect excess style, which closes the mythological system of the influencer.

On the other pole of food correctness is the Bulgarian influencer Sophie Yotova. She is not a vegan, she is not a fighter for animal rights and 
climate protection on the planet. Her passion and sacred beliefs are about the extraordinary role that a healthy and balanced diet can have on an individual's personal development. This applies not only to physical fitness, but also to much deeper levels of achieving existential harmony. Unlike Morenstein, she is very good with words and it is no coincidence that her influencer career began with a blog. ${ }^{3}$ She not only describes well what she truly believes, but illustrates it with direct references to her personal life and profound transformation. Her personal mythology spans two phases. As a child, she permanently lost her sense of smell, and at the same time, her grandmother was a strong influence in initiating her into the culinary arts despite this infirmity.

Initially Sophie chose a corporate career where she was very successful but at a high cost to herself. The world of business is a world of ostentation and image, a world that imposes false standards of physical fitness. Instead of being a source of balance, it can actually contribute additional stress of dieting and restrictions. For some time Sophie struggled with her appearance and weight through strict dieting, not realising and admitting to herself that in doing so she was sinking deeper into an eating disorder. It was then that she turned to the philosophy of healthy eating, which very soon took hold of her completely and led to a complete change in her life. She ditched her corporate career and devoted herself to her true passion and calling.

Sophie's Influencer mission starts from a desire to pass on the success of this life philosophy and passion to as many people as possible. If this can become her profession - all the better. In fact, it's all part of the narrative she uses to influence her followers. The philosophy of balance accompanies her every recipe and performance. Sophie is very creative in her content. She offers healthy recipes of simple yet very tasty dishes. "Balance" means not living in deprivation of something whose consumption would lead to excessive weight or other negative consequences. "Balance" means developing the ability to create food that satisfies all your taste requirements, but in such a way that it does not lead to undesirable consequences. Sophie's attitude infects her followers with the ability to amaze themselves every time they create unique culinary experiences with very simple ingredients.

\footnotetext{
3 “The blog is my baby, my materialized philosophy, my vocation and my soul in physical form". This is part of an interview with Sofi Yotova where is summarized everything essential for my analysis: https://woman.bg/dieti-i- fitnes/transformatsiyata-na-tova-momiche-ya-nauchi-da-zhivee.50885.html (accessed 18 August 2021).
} 
Success was not late in coming. Sophie developed a video channel and her own brand ${ }^{4}$. She holds paid workshops on creative cooking. A leading publishing house published her book of recipes, and she has been a regular guest on the leading TV shows in Bulgaria. Like Morenstein, she is true to herself and her sacred beliefs in every single manifestation. On the wave of the global trend for healthy eating, her mission is to impose a certain lifestyle, even culture, that gives much more meaning to food and nutrition than its physiological purposes.

\section{Conclusions}

Perhaps for many, the word influencer conjures up images of the thousands of semi-nude male and female beauties on Instagram who, in a much simpler way than the influencer system described above, have many more followers than my examples. If we force the theoretical framework, we can probably identify some semblance of these elements, but we certainly will not be able to appreciate the merits of the proposed model. Sex appeal has a no less primordial impact and drive than nutrition and pre-expressive facial trustworthiness. However, the key concept here is trust, and this is all we care about. I believe that the key difference between elementary sexual influencing and its more complex forms is precisely trust and the complex mechanism for achieving it which I have tried to model in this paper.

\section{References}

Bankov, K. 2018. Overview of Consumer Culture in Bulgaria: From Perestroika to Facebook. In Krasteva-Blagoeva, E. (ed.). Approaching Consumer Culture. Global Flows and Local Contexts. New York: Springer, 93-111.

Bankov, K. 2021. "Face and Trust: A Semiotic Inquiry into Influencers, Money, and Amygdala”. Sign Systems Studies, Vol. 50 (in press).

Barba, E. 1993. The Paper Canoe: A Guide to Theatre Anthropology. London: Routledge.

Colins, P. 1998. "Faces, Trust and the Amygdala”. Trends in Cognitive Sciences, Vol. 2, Issue 7, p. 238.

Floch, J.-M. 2001. Semiotics, Marketing and Communication. New York: Palgrave.

$\overline{4}$ https://www.foodieboulevard.bg/ (accessed 18 August 2021). 
Holland, N. N. 1967. "The "Willing Suspension of Disbelief" Revisited". Centennial Review, Vol. 11, Issue 1, 1-23.

Volli, U. 2016. Il contagio della metafora. In Marino, G., M. Thibault (eds.). Viralita. Lexia. Rivista di Semiotica, 25-26. Ariccia: Aracne editrice, 55-72.

Martineau, P. 2019 "The WIRED Guide to Influencers. Everything You Need to Know about Engagement, Power Likes, Sponcon, and Trust." WIRED Guides. Available at: https://www.wired.com/story/what-is-an-influencer/.

Vincent, L. 2002. Legendary Brands: Unleash the Power of Storytelling to Create a Winning Marketing Strategy. Chicago: Dearborn Trade Publishing. 